\title{
Hand hygiene - social network analysis of peer-identified and management-selected change agents
}

Yew Fong Lee ${ }^{1,2}$, Mary-Louise McLaws ${ }^{3 *}$, Loke Meng Ong ${ }^{4}$, Suraya Amir Husin², Hock Hin Chua ${ }^{5}$, See Yin Wong ${ }^{5}$, Didier Pittet $^{6}$ and Walter Zingg ${ }^{6}$

\begin{abstract}
Background: Hand hygiene compliance can be improved by strategies fostering collaborative efforts among healthcare workers (HCWs) through change agents. However, there is limited information about how change agents shape the social networks of work teams, and how this relates to organisational culture. The objectives of this study were to describe the influence of peer-identified change agents (PICAs) and management-selected change agents (MSCAs) on hand hygiene, perception of their leadership style by peers, and the role of the organisational culture in the process of hand hygiene promotion.
\end{abstract}

Methods: This study, stratified in pre-, during, and post-intervention periods, was conducted between February 2017 and March 2018 in two wards at a tertiary care hospital in Malaysia. Hand hygiene promotion was facilitated either by PICAs (study arm 1) or MSCAs (study arm 2), and the two wards were randomly allocated to one of the two interventions. Outcomes were: 1) perceived leadership styles of PICAs and MSCAs by staff, vocalised during question and answer sessions; 2) the social network connectedness and communication patterns between HCWs and change agents by applying social network analysis; and 3) hand hygiene leadership attributes obtained from HCWs in the post-intervention period by questionnaires.

Results: Hand hygiene compliance in study arm 1 and study arm 2 improved by from 48\% (95\% Cl: 44-53\%) to $66 \%(63-69 \%)$, and from 50\% (44-55\%) to 65\% (60-69\%), respectively. There was no significant difference between the two arms. Healthcare workers perceived that PICAs lead by example, while MSCAs applied an authoritarian topdown leadership style. The organisational culture of both wards was hierarchical, with little social interaction, but strong team cohesion. Position and networks of both PICAs and MSCAs were similar and generally weaker compared to the leaders who were nominated by HCWs in the post-intervention period. Healthcare workers on both wards perceived authoritative leadership to be the most desirable attribute for hand hygiene improvement.

Conclusion: Despite experiencing successful hand hygiene improvement from PICAs, HCWs expressed a preference for the existing top-down leadership structure. This highlights the limits of applying leadership models that are not supported by the local organisational culture.

Keywords: Hand hygiene, Alcohol-based handrub, Leadership, Social network analysis, Behavioural change, Multimodal strategy, Organizational culture

\footnotetext{
* Correspondence: m.mclaws@unsw.edu.au

${ }^{3}$ School of Public Health and Community Medicine, UNSW Medicine, UNSW

Sydney, Level 3 Samuels Building, Sydney, NSW 2052, Australia

Full list of author information is available at the end of the article
}

(c) The Author(s). 2019 Open Access This article is distributed under the terms of the Creative Commons Attribution 4.0 International License (http://creativecommons.org/licenses/by/4.0/), which permits unrestricted use, distribution, and reproduction in any medium, provided you give appropriate credit to the original author(s) and the source, provide a link to the Creative Commons license, and indicate if changes were made. The Creative Commons Public Domain Dedication waiver (http://creativecommons.org/publicdomain/zero/1.0/) applies to the data made available in this article, unless otherwise stated. 


\section{Background}

Globally, hundreds of millions of patients suffer from healthcare-associated infections every year with a higher burden in developing countries $[1,2]$. Hand hygiene has been accepted as one of the most cost-effective measures in reducing both cross-transmission of microorganisms and healthcare-associated infections. Despite evidence of its effectiveness, compliance with hand hygiene is rather low [3]. This may be due to the nature of hand hygiene being an action, driven by behaviour [4], and taking part within the socio-economical and organisational context of healthcare facilities $[5,6]$. Compliance is influenced by leadership engagement, peer pressure, and role modelling $[5,6]$. Improvement strategies often fail because of insufficient implementation skills [7], which need to take into account behavioural aspects as part of the organisational culture [8, 9]. Strategies fostering collaborative efforts and the development of partnership among healthcare workers (HCWs) have been shown to be beneficial in both hand hygiene promotion and the prevention of healthcare-associated infections [5, 6, 10-13]. Such interventions aim at influencing social networks within work teams and across healthcare professions with the final goal to change the organisational culture of an institution [14]. There is limited information about the way change agents shape the social networks of work teams in healthcare, and how this relates to organisational culture.

The objectives of this study were to describe the influence of peer-identified change agents (PICAs) and management-selected change agents (MSCAs) on hand hygiene, the perception of their leadership style by peers, their ability to shape team dynamics, and the role of the organisational culture in this process. This report is part of a study examining the effect of change agents on hand hygiene behaviour in acute healthcare (Lee YF et al. Hand hygiene promotion delivered by change agentsTwo attitudes, similar outcome, forthcoming).

\section{Methods}

This study was conducted in collaboration with the Malaysian Ministry of Health, the World Health Organization (WHO) Collaborating Centre on Patient Safety at the University of Geneva Hospitals (HUG), Switzerland, and the University of New South Wales Sydney (UNSW Sydney), Australia. Malaysia is an upper-middle-income country in Southeast Asia [15]. Sarawak is the largest of the 13 states in Malaysia and is situated on Borneo Island [16]. The state has the most diverse population in Malaysia, with more than 40 ethnic groups, and the highest percentage of Christians [15, 16]. In recent years, the economy has shifted away from traditional mining, agriculture and forestry towards high-tech industries with renewable energy. Sarawak has 23 public hospitals and special medical institutions. The total number of public and private hospital beds per 1000 people was 1.5 in 2015 [17] compared with the average for Malaysia of 1.8 in 2012 [18]. The doctor-to-population ratio in Sarawak was 1:1184 [17] compared with the Malaysian average of 1:632 in 2017 [18].

\section{Setting}

Between February 2017 and March 2018, two medical wards (study arm 1, study arm 2) were selected for an intervention on hand hygiene promotion at the Sarawak General Hospital, a university-affiliated, tertiary care hospital in Kuching, Malaysia. Kuching is the largest city and the economic centre in Sarawak with a total population of 325,132 [17]. The two wards were selected because of their interest, commitment and receptiveness towards improving hand hygiene behaviour (Lee YF et al. Hand hygiene promotion delivered by change agents-Two attitudes, similar outcome. forthcoming). Each ward had 42 official beds, with nurse-to-doctor ratios of 69:6 and 64:5, respectively. The nurse-to-patient ratio was 1: 2 in both wards. Both wards consisted of a mixed internal medicine adult patient population, often with chronic diseases and, in case of overflow in the intensive care unit, some patients underwent ventilation.

The study was stratified in pre-, during, and postintervention periods of 48-56 days (Lee YF et al. Hand hygiene promotion delivered by change agents-Two attitudes, similar outcome. forthcoming).

\section{Intervention}

Intervention, outcome measurement, and data entry are described in detail elsewhere (Lee YF et al. Hand hygiene promotion delivered by change agents-Two attitudes, similar outcome. forthcoming). In brief, before the pre-intervention period, HCWs in the two wards anonymously nominated and ranked 10 peers to become their change agents for hand hygiene promotion before the wards were randomly assigned to PICAs (study arm 1) or MSCAs (study arm 2). The nurse unit manager and the head of the medical department selected change agents for study arm 2 during the preintervention period. In total, six change agents, five nurses and 1 doctor, were selected for each study arm to assure that at least one change agent would be present on any study day. To reduce bias, no information on randomisation was revealed, and both PICAs and MSCAs were told that they were selected by senior management. Senior management refers to members of the medical and nursing boards of the department. The 6 PICAs in study arm 1 were HCWs with the highest numbers of nominations by their peers. Both PICAs and MSCAs were given the task to promote hand hygiene in their work teams during the intervention period by encouraging peers to perform hand hygiene, giving feedback, and offering correction or congratulation on missed or observed hand hygiene opportunities. 


\section{Outcome measurement}

Trained and validated auditors measured hand hygiene compliance by direct hand hygiene observation using the WHO methodology [19], and has been reported separately (Lee YF et al. Hand hygiene promotion delivered by change agents-Two attitudes, similar outcome. forthcoming). During the intervention period, the principal investigator conducted 8 walk-arounds in each study arm with the aim of observing interactions between change agents and staff and initiatives taken by the change agents to promote hand hygiene. In the post-intervention period, six question and answer (Q\&A) sessions in both English and Malaysian were organized to explore the opinion of nurses towards PICAs and MSCAs. The sessions were conducted over 6 days, with a maximum of 10 randomly allocated participants per session, in total 57 and 55 nurses in study arm 1 and study arm 2, respectively. Written informed consent was obtained from all participants. A local infection prevention and control expert facilitated the Q\&A sessions using a semi-structured interview guide that addressed five areas of leadership attributes: attitude, self confidence, approachability, team role, and decision-making capacities [5, 20]. The sessions were audio-recorded, transcribed verbatim and translated into English. In addition, the participating nurses re-nominated five peers (nurses only) they preferred as leaders for hand hygiene promotion. Doctors did not participate in this exercise. They also listed and ranked (as free text) three main leadership qualities they considered important for hand hygiene promotion.

\section{Social network analysis}

Data from the re-nomination lists were used to perform social network analysis with the NodeXLPro software (Social Media Foundation, Belmont, CA, USA) [21]. Three dimensions were distinguished: 1) visual sociograms of the entire network; 2) calculations of geodesic distance, density, and reciprocity of the network as a whole; 3) calculation of centrality of the five most nominated individuals. Sociograms visualize the position of staff within the post-intervention re-nomination network. Each HCW on the ward represents a 'node' and the lines between the nodes describe the personal network of the HCWs and the relational distance within this network. Geodesic distance measures the shortest route or pathway between two individuals and the range of their connections within the network. The distance is reported as an average and maximum distance between individuals $[22,23]$. Density measures the total interactions (or relationships) between individuals divided by the total possible interactions (relationships) between individuals within the network. Reciprocity measures the degree of reciprocal nominations between individuals (individuals nominating each other). Vertical hierarchies have low reciprocity (little nominations between individuals), while horizontal hierarchies have high reciprocity (many nominations between individuals). Centrality measures individuals who are most connected and as such will hold influential positions in the network [24]. The most common measures are degree centrality, closeness centrality, and betweenness centrality [25]. In the current study, degree centrality is the measurement of nominations directed towards an individual within the network [24, 25]. Closeness centrality is the average length of the shortest path between individuals. Closeness centrality captures how close one individual is to other individuals within the network, based on how quickly or easily each individual can interact with other individuals. Smaller numbers indicate shorter distance (easier interaction). Betweenness centrality measures the number of times an individual is on the shortest path between two other individuals. A high betweenness centrality indicates that an individual is an influential gatekeeper and is connected to the otherwise disconnected individuals in the network.

\section{Results \\ Hand hygiene compliance}

Compared to the pre-intervention period, hand hygiene compliance improved from $48 \%$ (95\% CI: 44-53\%) to $66 \%$ (63-69\%), and from 50\% (44-55\%) to 65\% (60-69\%) during the intervention period in study arm 1 and study arm 2 , respectively. There was no significant difference of hand hygiene improvement between the two study arms (Lee YF et al. Hand hygiene promotion delivered by change agents-Two attitudes, similar outcome. forthcoming).

\section{Question and answer sessions}

Perceived leadership styles of PICAs and MSCAs, expressed by HCWs during the Q\&A sessions in the post-intervention period, differed substantially between the two study arms and in all five areas of leadership attributes (Table 1). In accordance with observations during the ward walk-arounds, MSCAs were reported to apply an authoritative leadership style while PICAs acted more by being a role model.

A total of 69 and 68 leadership attributes for hand hygiene promotion were received from Q\&A participants of study arm 1 and study arm 2, with 201 and 172 citations, respectively. The most commonly quoted leadership quality for hand hygiene promotion was a "strict attitude", cited by $40 \mathrm{HCWs}(40 / 56 ; 70 \%)$ in study arm 1 and 27 times $(27 / 55 ; 49 \%)$ in study arm 2 (Table 2). 
Table 1 Perceived leadership styles of peer-identified change agents and management-selected change agents

\begin{tabular}{|c|c|c|}
\hline Leadership attributes & $\begin{array}{l}\text { Study arm } 1 \\
\text { Peer-identified change agents }\end{array}$ & $\begin{array}{l}\text { Study arm } 2 \\
\text { Management-selected change agents }\end{array}$ \\
\hline Attitude & $\begin{array}{l}\text { Not pushy, mostly easy-going; positive attitude; } \\
\text { motivated to improve hand hygiene practices } \\
\text { in the ward }\end{array}$ & $\begin{array}{l}\text { Very determined to improve hand hygiene } \\
\text { compliance; strong-minded; goal orientated }\end{array}$ \\
\hline Self-confidence & $\begin{array}{l}\text { Somewhat lacking self-confidence; concerns } \\
\text { about their leadership capacities }\end{array}$ & Very assertive \\
\hline Approachability & $\begin{array}{l}\text { Visible and always receptive; very friendly; not } \\
\text { strict; empathetic }\end{array}$ & $\begin{array}{l}\text { Approachable attitude, but sometimes unavailable } \\
\text { due to other activities; were strict; almost dictatorial }\end{array}$ \\
\hline Team role & $\begin{array}{l}\text { Strong team players; followed-through tasks with } \\
\text { other team members; always performed good } \\
\text { hand hygiene practices (good role models) }\end{array}$ & $\begin{array}{l}\text { Acted as team managers; kept their distance; } \\
\text { always performed good hand hygiene practices } \\
\text { (good role models) }\end{array}$ \\
\hline Decision-making capacities & Somewhat lacking & Prominent \\
\hline
\end{tabular}

\section{Social network analysis}

Social network analysis was performed on 56 and 55 HCWs in study arm1 and study arm 2, respectively. One HCW from study arm 1 did not participate in the social network questionnaire. Ward HCWs included senior, junior and auxiliary nurses with an average work experience of 7 years in study arm 1 and 6 years in study arm 2 , respectively. The wards were similar for the number of nominated relationships with 315 and 295 in study arm 1 and study arm 2, respectively. The sociograms illustrate similar social networks in both study arms (Fig. 1). Peer-identified change agents and MSCAs who were re-nominated in the post-intervention period had strong networks. One PICA (\#11) and one MSCA (\#60) who were nominated in the pre-intervention period but not re-nominated in the post-intervention period were less connected than other initially nominated HCWs. Figure 1 shows the visual sociograms of the entire network.

The average and maximum geodesic distances between individuals in both study arms were 1.9 and
3.0, respectively. On average, each $\mathrm{HCW}$ in both study arms was only two steps away from a renominated leader, indicating that both networks were cohesive. The density of both networks was 0.08 for each study arm, indicating that there was little interaction between HCWs in both networks. Reciprocities of study arm 1 and study arm 2 were low, 0.08 and 0.10 , respectively. This indicated a hierarchical network with only 8 and $10 \%$ of the pairs being nominated by each other.

The medians for the degree centrality, closeness centrality, and betweenness centrality of PICAs and MSCAs indicate that the leadership styles of both PICAs and MSCAs would equally influence hand hygiene (Table 3 ). Ranges of centrality were similar in both study arms.

The median centralities for the top five re-nominated leaders in the post-intervention period were similar in both study arms (Table 4). However, the range for the betweenness centrality in study arm 2 was wide (232.7-687.0), while this range in study arm 1 was narrow (234.3-387.8).

Table 2 Preferred leadership qualities for hand hygiene promotion cited by healthcare workers

\begin{tabular}{|c|c|c|c|c|c|c|c|}
\hline \multicolumn{4}{|c|}{ Study arm 1 (PICAs) } & \multicolumn{4}{|c|}{ Study arm 2 (MSCAs) } \\
\hline \multirow[t]{2}{*}{ Rank } & \multirow{2}{*}{$\begin{array}{l}\text { Preferred leadership } \\
\text { qualities }\end{array}$} & \multicolumn{2}{|c|}{ Frequency $(n=56 \mathrm{HCWs})$} & \multirow[t]{2}{*}{ Rank } & \multirow{2}{*}{$\begin{array}{l}\text { Preferred leadership } \\
\text { qualities }\end{array}$} & \multicolumn{2}{|c|}{ Frequency $(n=55 \mathrm{HCWs})$} \\
\hline & & $\bar{N}$ & $\%$ & & & $\bar{N}$ & $\%$ \\
\hline 1 & Strict & 40 & 71.4 & 1 & Strict & 27 & 49.1 \\
\hline 2 & Responsible & 29 & 51.8 & 2 & Good role model & 25 & 45.5 \\
\hline 3 & Hardworking & 14 & 25.0 & 3 & Always reminds & 15 & 27.3 \\
\hline 4 & Knowledgeable & 9 & 16.1 & 4 & Responsible & 14 & 25.5 \\
\hline 5 & Speaks up & 9 & 16.1 & 5 & Committed / dedicated & 12 & 21.8 \\
\hline 6 & Disciplined & 6 & 10.7 & 6 & Friendly & 9 & 16.4 \\
\hline 7 & Senior & 6 & 10.7 & 7 & Disciplined & 8 & 14.5 \\
\hline 8 & Soft spoken & 5 & 8.9 & 8 & Hardworking & 5 & 9.1 \\
\hline 9 & Honest & 5 & 8.9 & 9 & Good attitude & 5 & 9.1 \\
\hline 10 & Good teacher & 4 & 7.1 & 10 & Good teacher & 4 & 7.3 \\
\hline
\end{tabular}



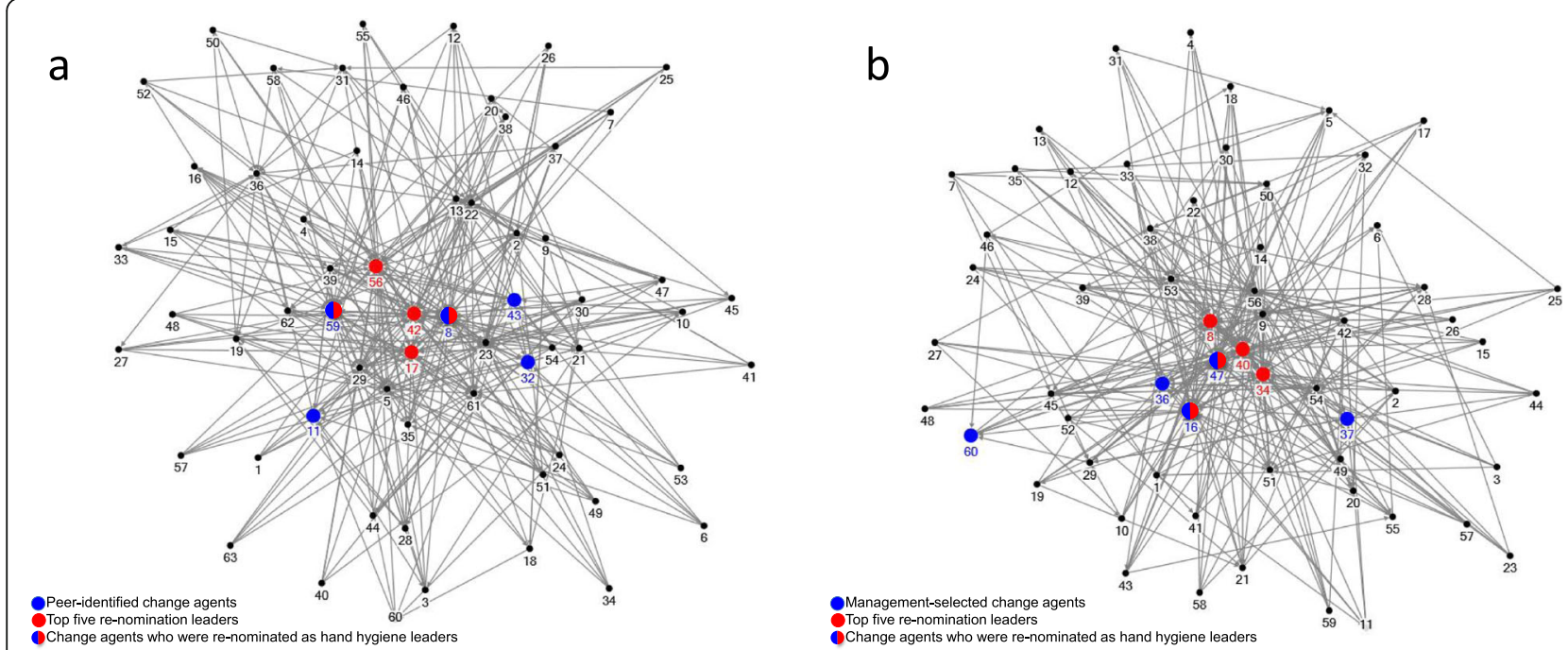

Fig. 1 Sociograms visualizing the positions of peer-identified and management-selected change agents (5 nurses each) within the re-nominated leaders. a Study arm 1 - Peer-identified change agents. b Study arm 2 - Management-selected change agents

Compared to PICAs, MSCAs had more work experience both overall and in the ward (Table 5). Both PICAs and MSCAs had low betweenness centralities compared to the top-five re-nominated leaders (Table 5).

\section{Discussion}

The fact that there was no difference in hand hygiene compliance between the two study arms was a major finding of the intervention strategy of the overall study (Lee YF et al. Hand hygiene promotion delivered by change agents-Two attitudes, similar outcome. forthcoming). Qualitative Q\&A sessions provided in-depth insight into perceptions of

Table 3 Centrality of the change agents nominated before the pre-intervention period

\begin{tabular}{llllll}
\hline & ID & $\begin{array}{l}\text { Change } \\
\text { Agent }\end{array}$ & & Centrality & \\
\cline { 5 - 6 } & & & Degree & Closeness & Betweenness \\
\hline Study arm 1 & 8 & Yes & 25 & 0.010 & 387.8 \\
PICAs & 59 & Yes & 18 & 0.010 & 234.3 \\
& 43 & Yes & 7 & 0.008 & 70.6 \\
& 11 & Yes & 7 & 0.008 & 46.7 \\
& 22 & Yes & 5 & 0.008 & 40.8 \\
Study arm 2 & 47 & Yes & 25 & 0.011 & 327.4 \\
MSCAs & 16 & Yes & 21 & 0.011 & 211.0 \\
& 36 & Yes & 12 & 0.010 & 86.5 \\
& 37 & Yes & 4 & 0.009 & 25.7 \\
& 60 & Yes & 3 & 0.009 & 18.8 \\
& Median & 12 & 0.010 & 86.5 \\
\hline
\end{tabular}

PICAs Peer-identified change agents, MSCAs Management- selected change agents leadership styles by HCWs, while quantitative social network analysis explained relationships of complex interactions in work teams. The methods complemented each other and provided a picture of socially cohesive work teams with staff nominating socially well-connected peers, while preferring the status-quo of a strict, even authoritarian, leadership style for hand hygiene promotion.

The little interaction between HCWs, the low number of re-nominated leaders $(2 / 5)$, and the low nominatornominee ties suggest that the networks in both study arms were highly hierarchical. The average short distance between the HCWs suggested that both work

Table 4 Centrality of the top five re-nominated leaders in the post-intervention period

\begin{tabular}{llllll}
\hline & ID & \multirow{2}{l}{$\begin{array}{l}\text { Change } \\
\text { Agent }\end{array}$} & & \multicolumn{2}{l}{ Centrality } \\
\cline { 5 - 6 } & & & Degree & Closeness & Betweenness \\
\hline Study arm 1 & 8 & Yes & 25 & 0.010 & 387.8 \\
PICAs & 17 & No & 23 & 0.010 & 371.7 \\
& 42 & No & 23 & 0.010 & 359.8 \\
& 56 & No & 24 & 0.010 & 315.0 \\
& 59 & Yes & 18 & 0.010 & 234.3 \\
Study arm 2 & 40 & No & 36 & 0.013 & 687.0 \\
MSCAs & 34 & No & 28 & 0.011 & 438.1 \\
& 47 & Yes & 25 & 0.011 & 327.4 \\
& 8 & No & 23 & 0.011 & 295.9 \\
& 16 & Yes & 21 & 0.011 & 232.7 \\
& Median & 25 & 0.011 & 327.4 \\
\hline
\end{tabular}

PICAs Peer-identified change agents, MSCAs Management- selected change agents 
Table 5 Work experience and centrality of change agents and the top-five re-nominated leaders

\begin{tabular}{|c|c|c|c|c|c|c|}
\hline & & \multirow{2}{*}{$\begin{array}{l}\text { Working years in } \\
\text { healthcare Median } \\
\text { (range) }\end{array}$} & \multirow{2}{*}{$\begin{array}{l}\text { Working years in } \\
\text { the ward Median } \\
\text { (range) }\end{array}$} & \multicolumn{3}{|c|}{ Centrality Median (range) } \\
\hline & & & & Degree & Closeness & Betweenness \\
\hline \multirow{2}{*}{$\begin{array}{l}\text { Study arm } 1 \\
\text { PICAs }\end{array}$} & Peer-identified change agents & $5(3-8)$ & $5(3-5)$ & $7(5-25)$ & $0.008(0.008-0.010)$ & $70.6(40.8-387.8)$ \\
\hline & Top five re-nominated leaders & $15(3-17)$ & $5(2-15)$ & $23(18-25)$ & $0.010(0.010-0.010)$ & $359.8(234.3-387.8)$ \\
\hline \multirow{2}{*}{$\begin{array}{l}\text { Study arm } 2 \\
\text { MSCAs }\end{array}$} & Management selected change agents & $19(10-24)$ & $8(2-10)$ & $12(3-25)$ & $0.010(0.009-0.011)$ & $86.5(18.8-327.4)$ \\
\hline & Top five re-nominated leaders & $14(6-24)$ & $3(2-9)$ & $25(21-36)$ & $0.011(0.011-0.013)$ & $327.4(232.7-687.0)$ \\
\hline
\end{tabular}

PICAs Peer-identified change agents, MSCAs Management- selected change agents

teams were cohesive. However, poor reciprocity and low ties (interactions) suggested that the HCWs did not rely on each other for assistance, at least not for hand hygiene.

Two of the five re-nominated leaders in both study arms had been identified as change agents. Their renomination supports the diffusion of innovation theory [26] hypothesis that change agents are early adopters who make change acceptable for their peers. In the context of facilitating the adoption of good hand hygiene practice behaviour, these change agents were important for linking disconnected HCWs towards hand hygiene practice.

The PICAs in study arm 1 promoted hand hygiene improvement through leading by example. However, HCWs in this study arm indicated that, when given a choice, they preferred authoritarian leadership, even though they also expressed unease with this leadership style, and they renominated rather socially skilled peers in the postintervention period. These findings exemplify the predominance of the existing organisational culture on behaviour, even if it causes cognitive dissonance [9]. This may have been shaped by the local culture of native tribes in Sarawak always to appoint elderly seniors to be leaders [27]. However, preference for hierarchical organization structures by HCWs has been reported also in other countries and in different healthcare settings [28-30].

Our study has limitations. First, concerns about confidentiality in the Q\&A sessions may have motivated $\mathrm{HCW}$ s to re-nominate leaders for hand hygiene promotion based on social expectations rather than their personal preference. Second, the study duration was relatively short to observe the full effect of a behavioural change intervention on organisational culture. A prolonged post-intervention period may have influenced both hand hygiene compliance and the perception of leadership style by staff. We hypothesise that in the long-term, the effect of PICAs on hand hygiene compliance may be superior compared to MSCAs, because HCWs feel more at ease working with them. Third, the limited, although significant, improvement of hand hygiene compliance exemplifies the reality given the short study periods and mandating internal change agents.

\section{Conclusion}

Despite experiencing successful hand hygiene improvement from PICAs who led by example, HCWs expressed a preference for the existing authoritarian leadership structure. This highlights the limits of applying leadership models that are not supported by the local organisational culture, and urges the need to repeat such study in other cultural settings. There was no difference in hand hygiene improvement between both study arms.

\section{Abbreviations}

HCWs: Healthcare workers; MSCAs: Management-selected change agents; PICAs: Peer-identified change agents; Q\&A: Question and Answer;

WHO: World Health Organization

\section{Acknowledgements}

We want to thank the Institute of Clinical Research, National Institute of Health, Ministry of Health, Malaysia, for funding this publication. We wish to thank the hand hygiene trainers of HUG for the organization of the local train-the-trainers workshop, and the Malaysian Ministry of Health for their continuously commitment in enhancing patient safety. In particular, we would like to thank the following colleagues for advice, support, and contribution to this study: Norjannah Abas, Catherine Valarie Alek, Elizabeth Awang, Nordiah Awang Jalil, Sugiwati Boret, Aldrick Bungan, En Sze Chan, Aaron Chiew, Zin Hing Chin, Manjan Dagang, Dayang Diun, Amilita Esseary, Alan Fong, Ni'mat Hamadan, Fatin Amalina Ibrahim, Kandong Jarop, Rufina Shantie Jep, Isma Iskandar Juperi, Ren Jye Kim, Molly Kudin, Kamarudin Lajim, Sie Ngo Lau, Marilyn Libin, Monica Martin, Zaidah Mat, Glorita Philip, Sheila Ivy Rasidin, Ellisa Reba, Whel Rosen, Suria Sayid Adris, Esah Serman, Irip Ubai, Mary William Ago, Suria Zaini. Finally, we thank the Director General of Health Malaysia for approving this publication.

\section{Authors' contributions}

The initial design was by MLM and developed with YFL, LMO, DP, SAH and WZ. SYW, HHC, YFL, SAH and DP contributed to data collection and intervention in the wards. YFL performed social network analysis under the supervision of MLM and WZ. YFL interpreted the data under the supervision of MLM and WZ. YFL performed all ward walk-arounds. YFL, HHC and SYW coordinated the Q\&A sessions. YFL, HHC and MLM wrote the initial draft of the manuscript and all authors contributed to the paper. All authors have read and approved the final manuscript.

\section{Funding}

The research did not receive any specific grant from funding agencies in the public, commercial or no-for-profit sectors. The publication fee was funded by the Institute of Clinical Research, National Institute of Health, Ministry of Health, Malaysia. This work was supported by the Infection Control Programme and WHO Collaborating Centre on Patient Safety (SPCI/WCC), University of Geneva Hospitals and Faculty of Medicine, Geneva, Switzerland; hand hygiene research activities at the SPCI/WCC are also supported by the Swiss National Science Foundation (grant no. 32003B_163262). 


\section{Availability of data and materials}

The datasets used and/or analysed during the current study are available from the corresponding author on reasonable request.

\section{Ethics approval and consent to participate}

Written informed consent was obtained from each participant. The study was approved by the Medical Research and Ethics Committee of the Malaysian Ministry of Health (Reference: (7) KKM/NIHSEC/P16-971 for NMRR16-763-30060 (IIR))

\section{Consent for publication}

Not applicable.

\section{Competing interests}

The authors declare that they have no competing interests

\section{Author details}

'Institute of Global Health, Faculty of Medicine, University of Geneva, Geneva, Switzerland. ${ }^{2}$ Ministry of Health, Putrajaya, Malaysia. ${ }^{3}$ School of Public Health and Community Medicine, UNSW Medicine, UNSW Sydney, Level 3 Samuels Building, Sydney, NSW 2052, Australia. ${ }^{4}$ Clinical Research Centre \& Department of Medicine, Hospital Pulau Pinang, Georgetown, Malaysia. ${ }^{5}$ Sarawak General Hospital, Kuching, Sarawak, Malaysia. Infection Control Programme and WHO Collaborating Centre on Patient Safety, University of Geneva Hospitals and Faculty of Medicine, Geneva, Switzerland.

Received: 29 August 2019 Accepted: 4 November 2019

Published online: 28 November 2019

\section{References}

1. Allegranzi B, Nejad SB, Combescure C, Graafmans W, Attar H, Donaldson L, et al. Burden of endemic health-care-associated infection in developing countries: systematic review and meta-analysis. Lancet. 2011;377:228-41.

2. Rosenthal VD. Health-care-associated infections in developing countries. Lancet. 2011;377:186-8.

3. World Health Organization. WHO | Evidence for Hand Hygiene Guidelines. (2009) https://www.who.int/gpsc/tools/fags/evidence_hand_hygiene/en/. Accessed 27 Aug 2019

4. Whitby M, McLaws ML, Ross MW. Why healthcare workers don't wash their hands: a behavioral explanation. Infect Control Hosp Epidemiol. 2006;27: 484-92.

5. Anderson D, Ackerman-Anderson LS. Beyond change management: how to achieve breakthrough results through conscious change leadership. 2nd ed: San Francisco: Pfeiffer; 2010.

6. Zingg W, Holmes A, Dettenkofer M, Goetting T, Secci F, Clack L, et al. Hospital organisation, management, and structure for prevention of healthcare-associated infection: a systematic review and expert consensus. Lancet Infect Dis. 2015:15:212-24.

7. Sladek RM, Bond MJ, Phillips PA. Why don't doctors wash their hands? A correlational study of thinking styles and hand hygiene. Am J Infect Control. 2008;36:399-406.

8. Whitby M, Pessoa-Silva CL, McLaws ML, Allegranzi B, Sax H, Larson E, et al. Behavioural considerations for hand hygiene practices: the basic building blocks. J Hosp Infect. 2007;65:1-8.

9. Kwok YL, Harris P, McLaws ML. Social cohesion: the missing factor required for successful hand hygiene program. Am J Infect Control. 2017;1:222-7.

10. Pittet $\mathrm{D}$, Hugonnet $\mathrm{S}$, Harbarth $\mathrm{S}$, et al. Effectiveness of a hospital-wide programme to improve compliance with hand hygiene. Lancet. 2000;356: 1307-12.

11. World Health Organization, WHO Guide to Implementation of the WHO Multimodal Hand Hygiene Improvement Strategy. (2009). https://apps.who. int/iris/handle/10665/70030. Accessed 27 Aug 2019.

12. Schweizer ML, Reisinger HS, Ohl M, Formanek MB, Blevins A, Ward MA, et al. Searching for an optimal hand hygiene bundle: a meta-analysis. Clin Infect Dis. 2014;58:248-59.

13. Luangasanatip N, Hongsuwan M, Limmathurotsakul D, Lubell Y, Lee AS, Harbarth S, et al. Comparative efficacy of interventions to promote hand hygiene in hospital: systematic review and network meta-analysis. BMJ. 2015;351:h3728.

14. Castro-Sánchez $\mathrm{E}_{1}$ Holmes A. International implementation of WHO's hand hygiene strategy. Lancet Infect Dis. 2013;13:824-5.
15. The World Bank. The World Bank in Malaysia. (2019) http://www.worldbank. org/en/country/malaysia. Accessed 27 Aug 2019.

16. Department of Statistics Malaysia Official Portal. Sarawak Government. (2019). https:/www.dosm.gov.my/v1/index.php?r=column/cone\&menu_id= cIJnWTITbWFHdmUwbmtSTE1EQStFZZ09. Accessed 27 Aug 2019.

17. Sarawak Government. Sarawak Facts \& Figures. (2017). http://sarawakfacts. sarawak.gov.my/modules/web/policy.php?clsid=\&polid=368. Accessed 27 Aug 2019.

18. The World Bank. Hospital beds (per 1,000 people) | Data. (2019). https://data. worldbank.org/indicator/SH.MED.BEDS.ZS?end=2014\&locations=MY\&start= 2009. Accessed 27 Aug 2019

19. World Health Organization. WHO | Hand hygiene monitoring and feedback. (2013). https:/www.who.int/gpsc/5may/monitoring_feedback/en/. Accessed 27 Aug 2019

20. Daly J, Jackson D, Mannix J, Davidson P. Hutchinson M. The importance of clinical leadership in the hospital setting. J Healthcare Leadersh. 2014;6:75.

21. NodeXL. Your Social Network Analysis Tool for Social Media. .(2016) https:// www.smrfoundation.org/nodexl/. Accessed 27 Aug 2019.

22. Hawe P, Webster C, Shiell A. A glossary of terms for navigating the field of social network analysis. J Epidemiol Community Health. 2004;58:971-5.

23. Farbey BA. Structural models: an introduction to the theory of directed graphs. J Oper Res Soc. 1966;17:202-3.

24. Freeman LC. Centrality in social networks conceptual clarification. Soc Networks. 1978;1:215-39.

25. Wasserman S, Faust K. Social network analysis : methods and applications: Cambridge: Cambridge University Press; 1994.

26. Rogers EM. Diffusion of innovations. 5th ed. New York: Free Press; 2003.

27. Damschroder LJ, Aron DC, Keith RE, Kirsh SR, Alexander JA, Lowery JC. Fostering implementation of health services research findings into practice: a consolidated framework for advancing implementation science. Implement Sci. 2009;4:50.

28. Creswick N, Westbrook Jl. Social network analysis of medication adviceseeking interactions among staff in an Australian hospital. Int J Med. 2010; 79:e116-25.

29. Cott C. "We decide, you carry it out": a social network analysis of multidisciplinary long-term care teams. Soc Sci Med. 1997;45:1411-21.

30. Scott J, Tallia A, Crosson JC, Orzano AJ, Stroebel C, DiCicco-Bloom B, et al. Social network analysis as an analytic tool for interaction patterns in primary care practices. Ann Fam Med. 2005;3:443-8.

\section{Publisher's Note}

Springer Nature remains neutral with regard to jurisdictional claims in published maps and institutional affiliations.

Ready to submit your research? Choose BMC and benefit from:

- fast, convenient online submission

- thorough peer review by experienced researchers in your field

- rapid publication on acceptance

- support for research data, including large and complex data types

- gold Open Access which fosters wider collaboration and increased citations

- maximum visibility for your research: over $100 \mathrm{M}$ website views per year

At $\mathrm{BMC}$, research is always in progress.

Learn more biomedcentral.com/submissions 